\title{
Lead Site CV6LL
}

National Cancer Institute

\section{Source}

National Cancer Institute. Lead Site CV6LL. NCI Thesaurus. Code C90406.

V1 electrode (+) placed in the 6th intercostal space on the left side of the thorax along a line parallel to the level of the point of the elbow. 